\title{
MODELS AND TECHNIQUES IN GEODETIC MONITORING OF TECTONIC DEFORMATIONS IN GREECE
}

\author{
Rossikopoulos D., Fotiou A., Pikridas C. and Bitharis S. \\ Aristotle University of Thessaloniki, Department of Geodesy and Surveying, rossi@topo.auth.gr, \\ afotiou@topo.auth.gr,cpik@topo.auth.gr, stylbith@gmail.com
}

\begin{abstract}
The assessment and interpretation of the geodetic results regarding the detection of possible spatial displacements and the deformation parameters have to be combined with a realistic geophysical model for the area. Usually, this study is carried out by fitting the geodetic data to a polynomial function, which is considered sufficient to describe adequately the deformation pattern. In terms of the computational steps needed, this polynomial fitting can be accomplished simultaneously by the analysis of the geodetic observations in a dynamic adjustment or non simultaneously in a sequential approach. The main goal of this article is to give a short description of the related methods and to present rigorous processing strategies for the analysis of GNSS observations from continuously permanent stations in order to create a modern and improved geodetic velocity field for Greece. Emphasis is given on the reference frame definition problem.

Keywords: Deformation measurements, frame definition problem, GNSS neworks, Greek velocity field.
\end{abstract}

\section{Introduction}

Various geodetic methods for the extraction of displacements and deformation parameters are recognized as useful techniques in many geophysical studies. Within the last fifty years a great number of geodetic applications have been presented in the literature. They are based on repeated observations from geodetic networks properly established in areas under investigation and the analysis of the results between different epochs by means of appropriate models. All these methods can be divided into three major categories according to the determination of: global scale movements, regional movements and local deformations due to seismic or volcanic activity.

Local networks that cover relatively small areas include triangulation-trilateration in 2-d, geometric leveling and GNSS networks. Furthermore, it is possible to process simultaneously in 3-d the previous types of networks and moreover to have a common analysis of geodetic and photogrammetric measurements or even a common analysis of geometrical geodetic measurements with measurements related to the gravitational field. These networks can monitor the deformations of the earth's crust due to seismic activity, landslides, movements of road and mine slopes, etc. Networks established for the volcano's activity monitoring in order to predict its eruption, belong also in this class. Combining measurements of GNSS systems, which record the geometric changes in the volcano area, with measurements of the gravity field, which record the geometric changes and signals of the movements and accumulations of magma beneath the volcano, provide important information about a possible upcoming eruption a few dozen years before it happens.

Regional networks that cover a part of a country or even a continent consist mainly of permanent GNSS stations with the aim to monitor the movements of plates in a continental scale and establish 
national datum reference frames for geodetic activities in every country. The velocities determined by geodetic methods reflect the relative motions of tectonic plates - sections of earth's crust bounded by faults - that extend to depths within the limits of the upper crust at least. These movements, where the first order effects of an earthquake deformation cycle has been removed, are smooth and usually described as rigid rotations of tectonic plates on the earth's surface.

Global networks for monitoring the earth and its changes in time include GNSS, SLR (Satellite Laser Ranging), LLR (Lunar Laser Ranging) and VLBI (Very Long Baseline Interferometry) stations. The object of such networks is the creation of a global conventional geocentric reference frame characterized by high precision and homogeneity in space and time and the continuous determination of GNSS satellite orbits. Since the location of global stations are changing in time due to geophysical and tectonic processes and these changes are perceived by means of modern observations, the parameter of time must be considered in the analysis of the observations. Along with the coordinates of geodetic stations in a reference epoch, their changes over time are also resulted, e. g. the velocity of their movements. Ignoring these positional variations serious errors are introduced both in the satellite orbits and subsequently in any position on the earth's surface but also in the assessment of deformation parameters and the velocity of the tectonic plates movement regardless of the type of a network.

The reference frame definition problem, known also as the datum problem or the zero order design problem, has received considerable attention since the pioneering work of Meissl (1965) and his famous "inner error theory". Meissl's method has been popularized by Blaha (1971) and Pope (1973) who developed a powerful method of evaluating the pseudoinverse matrix introducing the so called "solution space". The relation of various solutions to Meissl's inner constraints has been established by Baarda (1973) with the introduction of the so called S-transformation. Subsequently all methods have been described in full length by Grafarend and Schaffrin (1974), Pelzer (1974), van Mierlo (1980), Koch (1982), Teunissen (1985). This problem dominated the geodetic literature in the 70s although it remains still opportune in GNSS applications and in the assessment of geodetic data for the detection of displacements and the estimation of deformation parameters.

The assessment and interpretation of the geodetic results for the detection of possible spatial displacements and the deformation parameters have to be combined with a realistic geophysical model for the area under consideration. Usually, fitting geodetic data to a polynomial function is considered sufficient to describe the deformation pattern what it is mainly described in the present paper. In terms of the computational strategy needed, polynomial fitting can be accomplished by one of the following (Rossikopoulos, 2003; Dermanis, 2009): (i) simultaneously analysis of geodetic observations in a dynamic adjustment model, (ii) non simultaneously in a sequential approach of the dynamic adjustment, (iii) by a simple comparison of the results between any two epochs.

The main intention of the present paper is a short description of the methods used mainly in the analysis of satellite observations for studying the kinetic behavior of tectonic plates in Greece giving emphasis on the reference system definition problem, perhaps the most important problem as it concerns the estimability of unknown parameters. For example, a false choice of constraints in order to define the reference system could lead to a wrong picture of the movements of tectonic plates in a region. Depending on the kind and nature of the used geodetic data, e.g. original observations or derived coordinates from a network adjustment, the corresponding mathematical model may suffer from some specific problems, such as the inconsistency of the reference system or the existence of non-positive covariance matrices.

\section{The reference system definition problem}

The extended equations of observations are used in a dynamic treatment of temporal networks where the deformation of the network is considered and the measurements of all epochs take part in a simultaneously data process. Classical observations (angles, distances, height differences) or modern satellite observations (GNSS, VLBI, SLR, etc) can be analyzed. The linear observation equations for $m$ epochs are written in matrix form as 
where $\mathbf{x}_{\mathrm{o}}$ is the vector of coordinate corrections for the reference epoch, $\mathbf{u}$ the vector of differences (displacement vector), $\mathbf{A}$ and $\mathbf{B}$ coefficient matrices and $\mathbf{v}$ the vector of observational errors.

The deformation networks can be treated as independent and be solved with the help of minimum constraints that define the reference system of coordinates without affecting the geometric characteristics of the network as defined by the observations. The inner constraints

$\dot{\mathbf{E}} \mathbf{x}=\mathbf{0}, \ddot{\mathbf{E}} \mathbf{u}=\mathbf{0}$

are considered the best choice in view of the connection of a time varying network, i.e. transforming the different network epoch solutions in a common reference system, at least for local networks, as it is the Volvi network (Fotiou et al., 2003), where the local deformations must be separated from the broader movements of tectonic plates. Representing as $u$ the vector of the temporal changes of the coordinates of network stations, each component is given by

$$
\begin{aligned}
& u_{i}=x_{i}^{\prime}-x_{i}=x_{i}\left(t^{\prime}\right)-x_{i}(t) \\
& v_{i}=y_{i}^{\prime}-y_{i}=y_{i}\left(t^{\prime}\right)-y_{i}(t) \\
& w_{i}=z_{i}^{\prime}-z_{i}=z_{i}\left(t^{\prime}\right)-z_{i}(t)
\end{aligned}
$$

The inner constraints $\ddot{\mathbf{E}} \mathbf{u}=\mathbf{0}$ determine the center of the reference system keeping fixed the gravity center of the network, by defining as zero the sum of temporal coordinate variations

$$
\begin{aligned}
& \sum_{i=1}^{N} u_{i}=\sum_{i=1}^{N}\left(x_{i}^{\prime}-x_{i}\right)=0 \\
& \sum_{i=1}^{N} v_{i}=\sum_{i=1}^{N}\left(y_{i}^{\prime}-y_{i}\right)=0 \\
& \sum_{i=1}^{N} v_{i}=\sum_{i=1}^{N}\left(z_{i}^{\prime}-z_{i}\right)=0
\end{aligned}
$$

The constraints for the network orientation are defined by the conditions that no rotation has happened around the axes through the gravity center of the network

$$
\begin{aligned}
& \sum_{i=1}^{N}\left\{\begin{array}{lll}
z_{i} & v_{i}-y_{i} w_{i}
\end{array}=\sum_{i=1}^{N}\left\{z_{i}\left(y_{i}^{\prime}-y_{i}\right)-y_{i}\left(z_{i}^{\prime}-z_{i}\right)\right\}=0\right. \\
& \sum_{i=1}^{N}\left\{\begin{array}{lll}
x_{i} & w_{i}-z_{i} & u_{i}
\end{array}\right\}=\sum_{i=1}^{N}\left\{x_{i}\left(z_{i}^{\prime}-z_{i}\right)-z_{i}\left(x_{i}^{\prime}-x_{i}\right)\right\}=0 \\
& \sum_{i=1}^{N}\left\{y_{i} u_{i}-x_{i} v_{i}\right\}=\sum_{i=1}^{N}\left\{y_{i}\left(x_{i}^{\prime}-x_{i}\right)-x_{i}\left(y_{i}^{\prime}-y_{i}\right)\right\}=0
\end{aligned}
$$

They occur as a solution to discrete versus time-depended networks by applying the reset criterion of the relative angular momentum, known as Tisserand criterion in continuous networks. The scale of network in case it can't be determined by the observations, is introduced through the constraint

$$
\sum_{i=1}^{N}\left\{x_{i} u_{i}+y_{i} v_{i}+z_{i} w_{i}\right\}=\sum_{i=1}^{N}\left\{x_{i}\left(x_{i}^{\prime}-x_{i}\right)+y_{i}\left(y_{i}^{\prime}-y_{i}\right)+z_{i}\left(z_{i}^{\prime}-z_{i}\right)\right\}=0
$$

keeping constant the mean square length of the network over time.

According to the above, the matrices $\dot{\mathbf{E}}$ and $\ddot{\mathbf{E}}$ have the analytical form

$$
\mathbf{E}=\left[\begin{array}{llll}
\mathbf{E}_{1} & \mathbf{E}_{2} & \ldots & \mathbf{E}_{N}
\end{array}\right]
$$

where each sub-matrix $\mathbf{E}_{i}$ corresponding to the point $P_{i}$, is given by 
$\mathbf{E}_{i}=\left[\begin{array}{ccc}1 & 0 & 0 \\ 0 & 1 & 0 \\ 0 & 0 & 1 \\ 0 & z_{i} & -y_{i} \\ -z_{i} & 0 & x_{i} \\ y_{i} & -x_{i} & 0 \\ x_{i} & y_{i} & z_{i}\end{array}\right]$

Consequently in a time dependent geodetic network a "common" reference system for all epochs is needed. The definition of the reference system in each epoch is based on analogous to the Meissl constraints, which are introduced for the coordinate differences in time. This procedure that has been proposed by Pelzer (1971) for geodetic applications of deformation measurements, is a discrete approximation to the definition of the reference system under time-continues data (Dermanis, 2002). In the case of separate adjustments per epoch the constraints are incorporated as inner constraints on the unknown corrections of approximate coordinates, using common approximate coordinates for all epochs.

The inner constraints in a simultaneous adjustment of observations of all epochs are the corresponding process of the optimal fitting of the coordinates of any epoch to the corresponding coordinates at the reference epoch, applying the well known 2-d or 3-d similarity transformation. Applying the similarity transformation between coordinates at different epochs the effects of different reference system definitions in the separate adjustments of observations are eliminated. Other effect are also reduced such as the systematic errors of GNSS data that affect the positions of the network stations as scale errors and rotational errors around the axes of the observation reference system. Furthermore a comprehensive, stable, for the wide area of the network, movement of the crust is reduced, usually described as a rigid transformation. While in the case of plane, rigid motion of a plate is represented by two translations and a rotation, studying larger areas, where the analysis should be carried out on the surface of the ellipsoid, the equivalent rigid motion is a rotation around a fixed pole with constant angular velocity. The corresponding parameters of the rigid motion are either the three cartesian components of the rotation vector, commonly referred to as "Euler vector", or its spherical components. Of course, a threedimensional "rigid body" transformation may be applied instead of the Euler's pole technique as it is an equivalent solution. When initial information related to how the crust deforms is available, there are various ways to modify or extend the optimization criterion according to what was presented above, such as the generalization of inner constraints

$\mathbf{u}^{T} \mathbf{W} \mathbf{u}=\min$.

with "weight matrix" W determined according to the available information. The solution is given by means of the minimum constraints

$\ddot{\mathbf{H}} \mathbf{u}=\ddot{\mathbf{E}} \mathbf{W} \mathbf{u}=\mathbf{0}$

If all points of the network are involved in the inner constraints, the weight matrix $\mathbf{W}$ is the identity matrix ( $\mathbf{W}=\mathbf{I})$. In the case of partial inner constraints, where only some points are selected for the reference system definition, for example points that are considered to be immovable, then the elements of the diagonal of $\mathbf{W}$ that correspond to these points are 1 while all other elements are 0 . Generally matrix $\mathbf{W}$ introduces any information relevant to the parameters $\mathbf{x}$. By an appropriate choice of this matrix the various options, for solving the problem of the reference system definition in temporal networks, can be generalized and put in a common framework (Rossikopoulos, 2016), such as those given by Prescott (1981), Darby (1985), Segall and Matthews (1988) or by the various movement models such as those given by Solomon and Sleep (1974), Minster and Jordan (1978). For example, a choice for the reference system definition problem is to minimize the "movement" in a particular direction, through minimal constraints. This "outer coordinate solution" was given by Prescott (1981) in a specific application where the relative movement, and therefore the direction to minimize the temporal differences of the coordinates, should be parallel to the direction of the fault. Darby (1982) generalized the outer coordinate 
solution noting that the preferred direction can be different at different stations and in the "model coordinate solution" of Segall and Matthews (1988) the displacement residuals are made as small as possible for a subset of stations only, a case of partial inner constraints.

\section{The analytical approach in the estimation of deformations}

Displacements may be treated as independent deterministic unknown parameters where their relation to an underlying function is ignored. This approach has the advantage that it is free from any doubtful assumptions about the structure of the underlying function and in addition the results of the simultaneous adjustment of observations are equivalent to those of separate adjustment per epoch.

On the other hand, the contrast between the discrete nature of the information, provided by the geodetic measurements, and the continuous information required to describe the deformation, makes clear that an appropriate approach to the analysis of geodetic data, related to the analysis of crustal motion, should not be limited to the classic techniques of adjustment of observations and parameter estimation but it has to somehow incorporate a solution to the problem of indirect interpolation. Applying interpolation techniques is not only a mathematical necessity for the estimation of deformation in the observation points but also for the expansion of the information at any point in the tectonically active area, a topic of obvious importance in geophysics. The dependence of displacements on underlying functions can be taken into account in different ways. One of these usually used is to introduce a more or less empirical model for the function, which involves unknown parameters to be estimated from the adjustment of the observations. Typical choices of an empirical model are linear combinations of known base functions with unknown coefficients. Based on the analytical functions

$$
\mathbf{u}=\mathbf{F} \mathbf{a}
$$

which are used to smooth-out the differential motions and to represent the deformation model, the observation equations are written as

$$
\mathbf{b}=\mathbf{A} \mathbf{x}_{o}+\mathbf{B} \mathbf{F} \mathbf{a}+\mathbf{v}
$$

where $\mathbf{a}$ are unknown parameters and $\mathbf{F}$ the matrix with elements depending on the used base functions. For the system definition problem in the network adjustment, the minimal constraints are introduced in a sequential form. Firstly the datum for the reference epoch is defined by applying the inner constrains

$$
\dot{\mathbf{E}} \mathbf{x}_{o}=\mathbf{0}
$$

and afterwards the datum for any other epoch is defined by convenient minimal constraints. Although we use the extended model (12), we must minimize the norm

$\mathbf{u}^{T} \mathbf{u}=\min$.

of the displacements, instead of $\mathbf{a}^{\mathrm{T}} \mathbf{a}=$ min that would lead to the inner constraints $\tilde{\mathbf{E}} \mathbf{a}=\mathbf{0}$. This solution (14) corresponds to the best fitting of the coordinates of the various epochs to a reference epoch. The minimal constraints, which correspond to the optimum criterion (14) and satisfy the condition

$$
\mathbf{a}^{T} \mathbf{F}^{T} \mathbf{F} \mathbf{a}=\mathbf{a}^{T} \mathbf{R} \mathbf{a}=\min .
$$

have the form

$$
\tilde{\mathbf{H}} \mathbf{a}=\mathbf{0}
$$

where $\tilde{\mathbf{H}}=\widetilde{\mathbf{E}} \mathbf{R}=\ddot{\mathbf{E}} \mathbf{F}$

with $\tilde{\mathbf{E}}$ the inner constraints $\widetilde{\mathbf{E}} \mathbf{a}=\mathbf{0}$ matrix

$\widetilde{\mathbf{E}}=\ddot{\mathbf{E}} \mathbf{F}\left(\mathbf{F}^{T} \mathbf{F}\right)^{-1}$

and $\ddot{\mathbf{E}}$ is the inner constraints matrix $\ddot{\mathbf{E}} \mathbf{u}=\mathbf{0}$ for the network of $N$ points at epoch $t_{\alpha}$. The deformation model (11) can be a space model, a time model or a space-time model. In the first case, where the displacements are considered as dependent on space only, we have for a point $P_{i}$

$\mathbf{u}_{i}=\mathbf{F}\left(\mathbf{x}_{i}\right) \mathbf{a}$ 
with the unknown parameters a common for all points at the same epoch. The observations equations for all $(m)$ epochs are

$$
\begin{gathered}
\mathbf{b}_{o}=\mathbf{A}_{o} \mathbf{x}_{o}+\mathbf{v}_{o} \\
\vdots \\
\mathbf{b}_{\alpha}=\mathbf{A}_{\alpha} \mathbf{x}_{o}+\mathbf{A}_{\alpha} \mathbf{F}_{\alpha} \mathbf{a}_{\alpha}+\mathbf{v}_{\alpha} \\
\vdots \\
\mathbf{b}_{m}=\mathbf{A}_{m} \mathbf{x}_{o}+\mathbf{A}_{m} \mathbf{F}_{m} \mathbf{a}_{m}+\mathbf{v}_{m}
\end{gathered}
$$

Useful tools for creating analytic polynomial-type models of the form of the above relations result from the implementation methods of the theory of elasticity, as the deformation of the earth is associated with the classical case of continuous media mechanics. The deformation parameters in simultaneous adjustment of network observations of different epochs are usually treated as deterministic parameters, where the deformation of the entire network area or large part of it is considered homogeneous or it is treated combined with analytical interpolation methods. The datum for the reference epoch is defined by applying the inner constrains

$$
\dot{\mathbf{E}} \mathbf{x}_{o}=\mathbf{0}
$$

and the datum for any other epoch is defined by the minimal constraints

$$
\tilde{\mathbf{H}}_{\alpha} \mathbf{a}_{\alpha}=\mathbf{0}
$$

where $\tilde{\mathbf{H}}_{\alpha}=\tilde{\mathbf{E}}_{\alpha} \mathbf{R}_{\alpha}=\ddot{\mathbf{E}}_{\alpha} \mathbf{F}_{\alpha}=\sum_{i=1}^{N} \ddot{\mathbf{E}}_{i} \mathbf{F}_{i}$

with $\mathbf{R}_{\alpha}=\mathbf{F}_{\alpha}^{T} \mathbf{F}_{\alpha}$ and $\widetilde{\mathbf{E}}_{\alpha}=\ddot{\mathbf{E}}_{\alpha} \mathbf{F}_{\alpha}\left(\mathbf{F}_{\alpha}^{T} \mathbf{F}_{\alpha}\right)^{-1}$

where $\ddot{\mathbf{E}}_{\alpha}=\left[\begin{array}{lll}\ddot{\mathbf{E}}_{1} & \ddot{\mathbf{E}}_{2} \ldots \ddot{\mathbf{E}}_{N}\end{array}\right]$ is the inner constraints matrix $\ddot{\mathbf{E}}_{\alpha} \mathbf{x}_{\alpha}=\mathbf{0}$ for the network at epoch $t_{\alpha}$.

Based on the above, the selection of matrix $\mathbf{R}_{\alpha}$ depends on the choice of the minimum constraints. These constraints are selected on the basis to minimize the uncertainty of the elements of the vector $\mathbf{u}_{\alpha}$, due to the different definition of the reference system of the network in each epoch. This uncertainty is minimized for each epoch of measurements through the condition $\mathbf{u}_{\alpha}^{T} \mathbf{u}_{\alpha}=\min$, which corresponds to the best fitting of the coordinates at the various epochs to the reference epoch. In equations (20) the deformation process was seen as a change from an initial to a final state of the continuous media without considering the time elapsed between the epochs. However, in many cases it is important to know the rate at which these changes occur, especially when we have a continuous data stream and the deformation is smooth in time. Examples of such applications were given by Morgan (1973), Bibby $(1975,1982)$ and Welsch (1986). In case the displacements are considered dependent on time only, the deformation model has the simple form

$$
\mathbf{u}_{i}=\mathbf{F}\left(t, t^{\prime}\right) \mathbf{a}_{i}
$$

If the movements are linear in time, we have the simple "velocity model"

$$
\mathbf{u}=\delta t_{\alpha} \dot{\mathbf{u}}
$$

where $\dot{\mathbf{u}}$ is the velocities vector and $\delta t_{\alpha}=t_{\alpha}-t_{o}$ (or $\delta t_{\alpha}=t_{\alpha}-t_{\alpha-1}$ ) the time difference. The first application of this velocity model was presented by Morgan (1973). Examples were given in Papo and Perelmuter (1983), Welsch (1986) and for vertical networks Vanicek et al. (1979), Mälzer et al. (1979). The observation equations for the $m$ epochs are written as

$$
\begin{gathered}
\mathbf{b}_{o}=\mathbf{A}_{o} \mathbf{x}_{o}+\mathbf{v}_{o} \\
\vdots \\
\mathbf{b}_{\alpha}=\mathbf{A}_{\alpha} \mathbf{x}_{o}+\mathbf{A}_{\alpha} \mathbf{F}_{\alpha} \mathbf{a}+\mathbf{v}_{\alpha}
\end{gathered}
$$


$\mathbf{b}_{m}=\mathbf{A}_{m} \mathbf{x}_{o}+\mathbf{A}_{m} \mathbf{F}_{m} \mathbf{a}+\mathbf{v}_{m}$

where the unknown parameters a are common for all epochs at the same point. When discontinuities occur between epochs $t_{o}$ and $t_{\alpha}$, the solution becomes significantly complicated. Movements are divided into long period, considered normal and occurre between the epochs of discontinuities (e.g. epoch of a seismic event), and episodic occurring during the earthquakes activity. In such cases, the approaches can be grouped as follows:

- The simultaneous adjustment must be applied only for epochs between two "temporal discontinuities".

- The causes of the temporal discontinuities can be included in the analytical models. In this way we have analytical functions describing the long period movements between discontinuities and also functions for the episodic movements derived from the cause of discontinuity.

With reference to the epoch of measurements we should note that:

- The reference epoch is not necessarily a measuring epoch.

- There is no need for observations to refer to a "network measurement epoch" as every measurement is related to its own epoch.

The datum definition constraints are written as

$\dot{\mathbf{E}} \mathbf{x}_{o}=\mathbf{0}, \tilde{\mathbf{H}} \mathbf{a}=\mathbf{0}$

where $\dot{\mathbf{E}}$ is the inner constraints matrix for the reference epoch and $\tilde{\mathbf{H}}$ selected in order to minimize the norm

$\mathbf{u}^{T} \mathbf{u}=\sum_{\alpha=1}^{m} \mathbf{u}_{\alpha}^{T} \mathbf{u}_{\alpha}=\mathbf{a}^{T}\left(\sum_{\alpha=1}^{m} \mathbf{F}_{\alpha}^{T} \mathbf{F}_{\alpha}\right) \mathbf{a}=\mathbf{a}^{T} \mathbf{R} \mathbf{a}=\min$.

given by $\tilde{\mathbf{H}}=\tilde{\mathbf{E}} \mathbf{R}=\ddot{\mathbf{E}} \sum_{\alpha=1}^{m} \mathbf{F}_{\alpha}$ where the inner constraints $(\widetilde{\mathbf{E}} \mathbf{a}=\mathbf{0})$ matrix is

$\widetilde{\mathbf{E}}=\ddot{\mathbf{E}}\left(\sum_{\alpha=1}^{m} \mathbf{F}_{\alpha}\right)\left(\sum_{\alpha=1}^{m} \mathbf{F}_{\alpha}^{T} \mathbf{F}_{\alpha}\right)^{-1}$

For the velocity model $\mathbf{u}=\delta t_{\alpha} \dot{\mathbf{u}}$ equation (28) becomes

$\tilde{\mathbf{H}} \mathbf{a}=\left(\sum_{\alpha=1}^{m} \delta t_{\alpha}\right) \ddot{\mathbf{E}} \dot{\mathbf{u}}=\mathbf{0}$ or $\ddot{\mathbf{E}} \dot{\mathbf{u}}=\mathbf{0}$

If the displacements are considered to be dependent on space and time, the analytical deformation model takes the form

$\mathbf{u}_{i}=\mathbf{F}\left(\mathbf{x}_{i}, t, t^{\prime}\right) \mathbf{a}$

where the unknown parameters a are common for all epochs and all points and the observations equations for the $m$ epochs have the same form as in (27). Examples are given in Bibby (1982), Snay et al. (1983, strain rate model) and Welsch (1986). The simultaneous adjustment of the observations of many epochs was presented by Whitten (1967), where movements are approached by functions of space and time of the above form. Subsequently relevant papers were given by Snay and Gergen (1978), Snay et al. (1983), Chrzanowski et al. (1986), Welsch (1986) and regarding the determination of the vertical movements by Holdahl (1978, 1980), Vanicek (1975), Vanicek et al. (1979), Holdahl and Hardy (1979) and Mälzer et al. (1979).

As in the previous case of dependent movements in time, the following should be noted:

- The reference epoch is not necessarily a measuring epoch.

- There is no need for observations to refer to a "network measurement epoch" as every measurement is related to its own epoch.

The minimal constrains which satisfy the condition $\mathbf{u}^{T} \mathbf{u}$ for all epochs are written 
$\tilde{\mathbf{H}} \mathbf{a}=\left[\sum_{i=1}^{N} \dot{\mathbf{E}}^{i}\left(\sum_{\alpha=1}^{m} \mathbf{F}_{\alpha}^{i}\right)\right] \mathbf{a}=\mathbf{0}$

where $\dot{\mathbf{E}}^{i}$ is the sub- matrix of $\dot{\mathbf{E}}$ corresponding to point $P_{i}$ and $\mathbf{F}_{\alpha}^{\mathrm{i}}$ the sub-matrix of $\mathbf{F}_{\alpha}$.

\section{The sequential solution}

The simplification of the algorithms implementing the above simultaneous adjustment of temporal observations lead to separate adjustment of observations, where specific algorithms are applied giving the same results as those of a simultaneous adjustment. These algorithms can be applied according to the type of available data. For example when the coordinates of the network stations and their covariance matrix are given for each epoch, they are considered as new (synthetic) observations. Generally, the analysis follows the steps:

a. Separate or single adjustment per epoch: This step includes the adjustment of the observations at each epoch, the statistical analysis and the final estimation of the coordinate set and its full covariance matrix at each epoch.

b. Best fitting of the coordinates at the various epochs to a reference epoch: The elimination of the difference between the coordinates at two distinct epochs, which is due to their different datum definition, is obtained by the optimal fitting of the coordinates of any epoch to the corresponding coordinates at the reference epoch, applying the well known 2-d or 3-d similarity transformation.

c. Adjustment with a deformation model: The coordinates at the reference epoch and the transformed ones as described in the second step are adjusted taking into account a deformation model. The mathematical model for all epochs is written in matrix form

$$
\begin{aligned}
& \mathbf{x}_{1}=\mathbf{x}_{o}+\mathbf{F}_{1} \mathbf{a}+\mathbf{v}_{1} \\
& \quad \vdots \\
& \mathbf{x}_{\alpha}=\mathbf{x}_{o}+\mathbf{F}_{\alpha} \mathbf{a}+\mathbf{v}_{\alpha} \\
& \quad \vdots \\
& \mathbf{x}_{m}=\mathbf{x}_{o}+\mathbf{F}_{m} \mathbf{a}+\mathbf{v}_{m} \\
& \text { or } \quad \mathbf{x}=\mathbf{x}_{o}+\mathbf{F} \mathbf{a}+\mathbf{v} \text { and } \mathbf{v}^{T} \mathbf{W} \mathbf{v}=\sum_{\alpha=1}^{m} \mathbf{v}_{\alpha}^{T} \mathbf{W}_{\alpha} \mathbf{v}_{\alpha}=\min .
\end{aligned}
$$

where $\mathbf{x}_{o}$ is the vector of coordinates for the reference epoch and with the term $\mathbf{F a}$ to describes the displacement vector $\mathbf{u}$. The displacements can be considered as dependent in time or in space and time. For the time span of the analysis period (all epochs), the corresponding covariance matrices $\mathbf{Q}$ derived from the transformations, are semi-positive definite. In this case, any choice of the generalized inverse matrix $\mathbf{W}=\mathbf{Q}^{-}$as weight matrix, and therefore the pseudo inverse matrix $\mathbf{W}=\mathbf{Q}^{+}$, leads to the best unbiased estimations for parameters $\mathbf{u}$.

The method is illustrated by Rossikopoulos et al. (1998), where GPS measurements are used associated to a 9-point geodetic network connecting the Greek and Italian coasts in the Ionian and Adriatic Sea. Other techniques for implementing the method of least squares for the adjustment of time-depended networks are presented in the literature, such as the Kalman filters, where the equation $\mathbf{u}=\mathbf{F} \mathbf{a}$ is the dynamic system that connects the epochs of measurements (Floyd at al. 2010), or other partitioned algorithms for the simultaneously adjustment of the temporal observations in a sequential mode (Rossikopoulos, 2016).

\section{From discrete to continuous information}

The fact that the analysis of the measurements provides only a discrete character, as a result of the discrete nature of the network stations, can be considered a disadvantage to the study of deformations using geodetic methods. In contrast the geophysical significant quantities have a continuous nature, 
because they are dependent on what is happening in a very small area around each point of the under studying area. For the extension of the information derived from the analysis of network measurements at each point in the area, it is necessary to apply interpolation methods in order to provide continuous information from discrete geodetic results. Of course, these disadvantages tend to disappear today, as the technology of three-dimensional scanners develops rapidly, both in terrestrial and space applications giving continuous information.

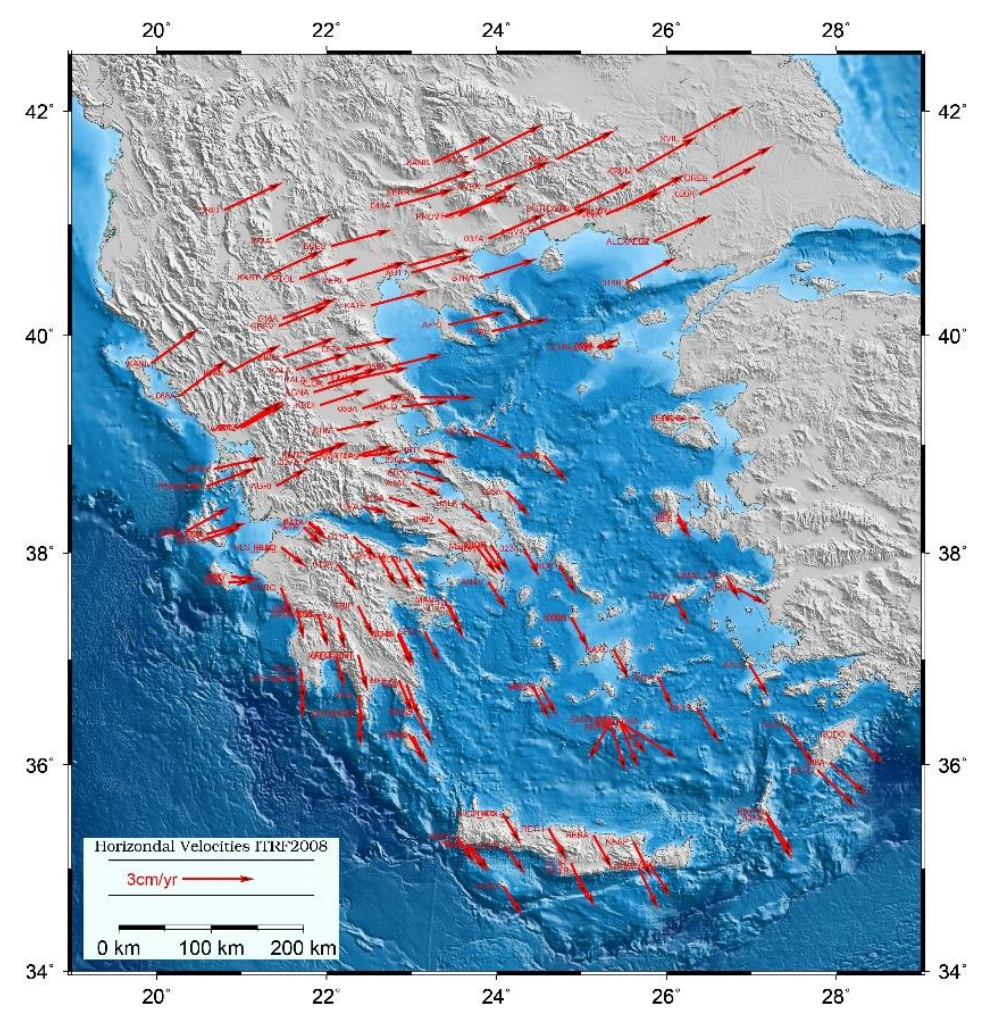

Figure 1 - Horizontal velocities relative to ITRF2008 (Bitharis et al. 2015).

Therefore the estimation of movements or the velocities or the geophysical parameters that can be derived from these, at any point in the area covered by the control network, after the connection of its temporal forms, interpolation methods are applied, where the area is divided into equal tectonic deformation regions and an analytical function for each region, generally in polynomial form, is fitted at temporal variations of the coordinates. A related method of analytical interpolation is that of piece-wise polynomials or splines with smoothness conditions imposed at the boundaries of their domains. Spline interpolation has been used by Bitharis et al. (2015), using bi-cubic splines for the interpolation of velocities of 155 GNSS permanent stations in Greece (Fig. 1), at the nodes of an appropriately selected grid. The continuous Greek geodetic velocity model relative to ITRF2008 is shown in figure 2.

The station coordinates and velocities depicted in figure 1, were estimated by a Kalman filtering sequential approach. The connection of the temporal forms of the network was made through their integration into the global reference system ITRF2008, which was conducted by means of the 7parameter similarity transformation for each epoch, using only IGS stations of the European part of the studied Greek Continuous Satellite Network (GCSNet), excluding the Greek stations.

In addition to the "analytical approach" of movements, developed in this paper as a part of adjustment algorithms, another way is to express the known limitations of the unknown functions of the movements through a covariance function, which describes the statistical behavior of its values, by the method of least squares collocation. 


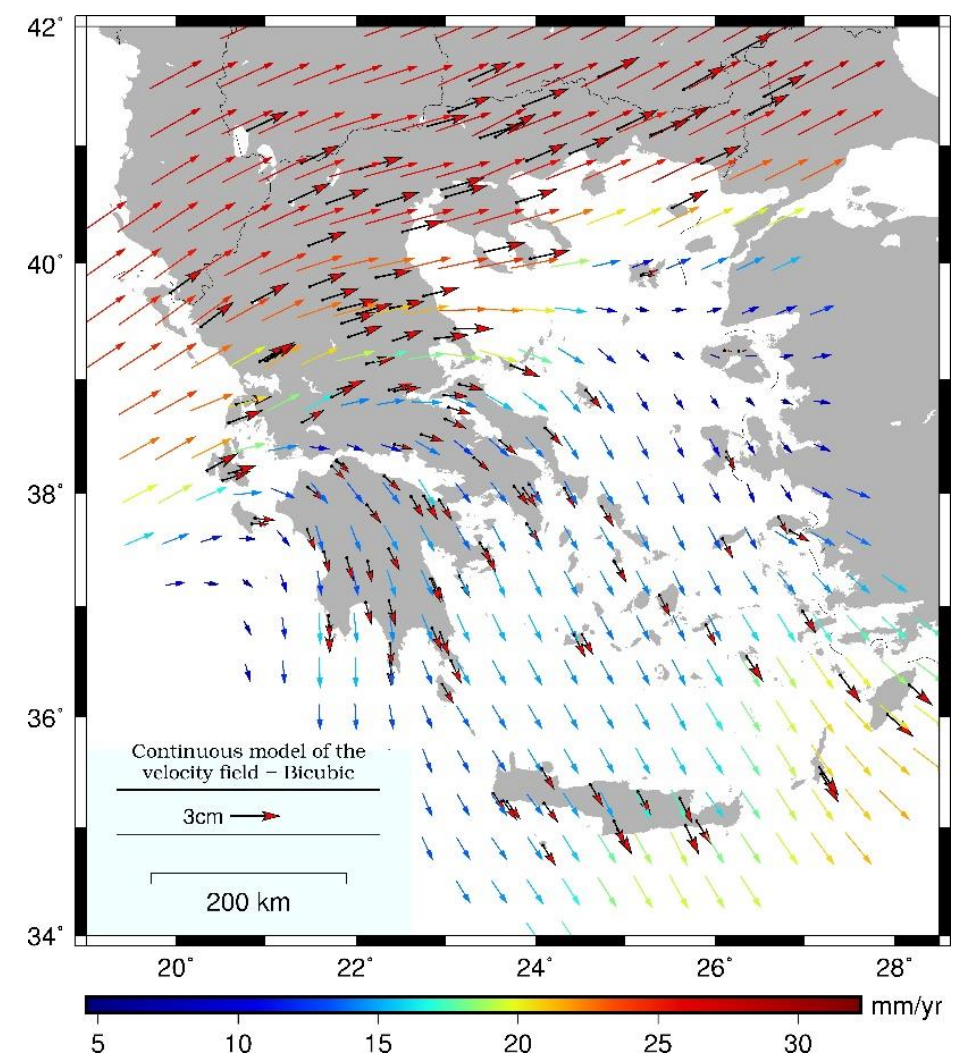

Figure 2 - Continuous Greek geodetic velocity model relative to ITRF2008 (Bitharis et al. 2015).

The least squares collocation is a generalized estimation method developed approximately in the 70s in order to analyze data of the gravitational field of the earth. Shortly after the method was applied to other scientific problems, for the analysis of observations related to quantities that depend on an unknown function. Collocation was first applied to the analysis of geodetic data for the calculation of strain parameters at the nodes of a grid in the area of Volvi in Greece (Dermanis et al., 1981) using the temporal variations of the network stations and in the area of Friuli in Italy (Bencicni et al., 1982). Applications to the estimation of the vertical movements were given by Hein and Kistermann (1981), Kanngieser (1983) and El-Fiky et al. (1997). Papers on horizontal and vertical movements (El-Fiky and Kato, 1999; Wu et al., 2006; Kahle et all., 1995; Chatzinikos et al., 2013), were followed.

Besides the analytical interpolation methods and least squares collocation, other methods that could be applied to generalize any quantity resulting from the temporal variations of network stations are given in the literature, such as pure numerical method of moving average or the method of finite elements, which is the most popular in geodetic literature due to its simplicity in how it is applied.

\section{References}

Baarda, W., 1973. S-Transformation and Criterion Matrices, Netherlands Geodetic Commision, Pulications on Geodesy, New Series, 5(1).

Bencini, P., Dermanis, A., Livieratos, E. and Rossikopoulos, D., 1982. Crustal Deformation at the Friuli Area from Discrete and Continuous Geodetic Prediction Techniques, Boll. di Geod. Sci. Aff., XLI, 137-148.

Bibby, H.M., 1975. Crustal Strain from Triangulation in Marlborough, New Zealand, Tectonophysics, 29, 529-540.

Bibby, H.M., 1982. Unbiased estimate of strain from triangulation data using the method of simultaneous reduction, Tectonophysics, 82, 161-174. 
Bitharis, S., Fotiou A., Pikridas C. and Rossikopoulos, D., 2015. A new crustal velocity field of Greece based on seven years (2008-2014) continuously operating GPS station data, Presented at the 26th IUGG General Assembly, June 22-July 2, Prague.

Blaha, G., 1971. Inner Adjustment Constraints with Emphasis on Range Observations. Department of Geodetic Science, Report No. 148, The Ohio State University.

Brunner, F.K., 1979. On the analysis of geodetic networks for the determination of the incremental strain tensor, Survey Review, XXV, 192, 56-67.

Brunner, F.K., Coleman, R. and Hirsch, B., 1981. A comparison of computation methods for crustal strains from geodetic measurements, Tectonophysics, 71, 281-298.

Caspary, W.F., 1987. Concepts of Network and Deformation Analysis, University of New South Wales, Kensington, Australia, 183 pp.

Chatzinikos, M., Fotiou, A., Pikridas, C.and Rossikopoulos, D., 2013. The Realization of a SemiKinematic Datum in Greece Including a New Velocity Model. In: Rizos, C. and Willis, P., eds., IAG 150 Years (1-9), Springer Berlin Heidelberg. http://doi.org/10.1007/1345_2015_93.

Chrzanowski, A., Chen, Y.Q. and Secord, J.M., 1983. On the strain analysis of tectonic movements using fault crossing geodetic surveys, Tectonophysics, 97, 297-315.

Darby, D., 1982. The analysis of variance of an underdetermined geodetic displacement problem, Geophys. Res. Lett., 9, 641-644.

Dermanis, A., 1985b. The role of frame definitions in the geodetic determination of crustal deformation parameters, Bulletin Géodésique, 59, 247-274.

Dermanis, A., 2002. The rank deficiency in estimation theory and the definition of reference frames. Presented at the V Hotine-Marussi Symposium on Mathematical Geodesy, June 12-21, Matera, Italy.

Dermanis, A., 2009. The Evolution of geodetic methods for the determination of strain parameters for earth crust deformation. In: Arabelos, D., Contadakis, M., Kaltsikis, Ch. and Spatalas, S., eds., Terrestrial and Stellar Environment, Volume in honor of Prof. G. Asteriadis. Publication of the School of Rural \& Surveying Engineering, Aristotle University of Thessaloniki, 107-144 pp.

Dermanis, A. and Rossikopoulos, D., 1988. Modeling Alternatives in Four-dimensional Geodesy, Pres. at the Int. Symp. Instumentation, Theory and Analyses for Integrated Geodesy, Sopron, May 16 to 20 .

Dermanis, A., Livieratos, E., Rossikopoulos, D. and Vlachos, D., 1981. Geodetic prediction of crustal deformations at the seismic area of Volvi, In: Deutsche Geod. Komm., Reihe B, 258(V), 234-248.

El-Fiky, G.S., Kato, T. and Fujii, Y., 1997. Distribution of the vertical crustal movement rates in the Tohoku district, Japan, predicted by least-squares collocation, J. Geodesy, 71, 432-442.

El-Fiky, G.S. and Kato, T., 1999. Continuous distribution of the horizontal strain in the Tohoku district, Japan, predicted by least-squares collocation, J. Geodynamics, 27, 213-236.

Floyd, M.A., Billiris, H., Paradissis, D., Veis, G., Avallone, A., Briole, P., McClusky, S., Nocquet, J.M., Palamartchouk, K., Parsons, B. and England, P., 2010. A new velocity field for Greece: Implications for the kinematics and dynamics of the Aegean, J. of Geoph. Res., 115, b10403, doi: 10.1029/2009JB007040.

Fotiou, A., Kagiadakis, V., Pikridas, C. and Rossikopoulos, D., 2003. Geodetically derived displacements and crustal deformation analysis: Application in the Volvi area, Proceedings of the 11th Int. Sym. on Def. Meas., FIG commission 6, Santorini, Greece, 25-28 May.

Frank, F.C., 1966. Deduction of Earth Strains from Survey Data, Bul. Seism. Soc. Amer., 56, 35-42.

Grafarend, E., 1985. Criterion matrices for deforming networks. In: Grafarend, E. and Sanso, F., eds., Optimization and Design of Geodetic Networks, Springer Verlag, 363-428.

Grafarend, E. and Schaffrin, B., 1974. Unbiased Free Net Adjustment, Survey Review, XXII/171, 200-218.

Heck, B., 1985. Ein- und zweidimensionale Ausreißertests bei der ebenen Helmert-Transformation, Zeitsschrift für Vermessungswesen, 10, 461-471.

Hein, G. and Kistermann, R., 1981. Mathematical foundation of non-tectonic effects in geodetic recent crustal movement models, Tectonophysics, 71, 315-334.

Holdahl, S.H., 1978. Models for extracting vertical crustal movements from leveling data, Int. Symp. on the Applications of Geodesy to Geodynamics, Columbus, Ohio. Department of Geodetic 
Science, Rep. No. 280, The Ohio State University, 183-190.

Holdahl, S.H., 1980. Crustal movements and Redefinition of Heights, Proc. of NAD Symp. 1980, Ottawa, 567-578.

Holdahl, S.H., 1982. Recomputations of vertical crustal motions near Palmdale, Califonia, 19591975, Journal of Geophysical Research, 87(B11), 9374-9388.

Holdahl, S.R. and Hardy, R.L., 1979. Solvability and multiquadric analysis as applied to investigations of vertical crustal movements, Tectonophysics, 52, 139-155.

Kahle, H.-G., Muller, M.V., Geiger, A., Danuser, G., Mueller, S., Veis, G., Billiris, H. and Paradissis, D., 1995. The strain field in northwestern Greece and the Ionian Islands: results inferred from GPS measurements, Tectonophysics, 249, 41-52.

Kanngieser, E., 1983. Application of Least-Squares Collocation to Gravity and Height Variations Associated with a Recent Rifting Process, Tectonophysics, 97, 265-277.

Malzer, H., Schmitt, G. and Zipplet, K., 1979. Recent Vertical Movements and their Determination in the Rhenish Massif, Tectonophysics, 53, 167-176.

Margrave, G.F. and Nyland, E., 1980. Strain from repeated geodetic surveys by generaized inverse methods, Can. J. Earth Sci, 17, 1020-1030.

Meissl, P., 1965. Ober die innere Genauigkeit dreidimensionaler Punkthaufen, Zeitsschrift für Vermessungswesen, 90(4), 109-118.

Meissl, P., 1969. Zusammenfassung und Ausbau der inneren Fehlertheorie eines Punkthaufens, Deutsche Geod. Komm. Reihe A, 61, 8-21.

Morgan, P., 1973. Crustal Velocity and Strain. In: Angus-Leppan, P.V., Bomford, A.G., Dooley, J.C. and Mather, R.S., eds., The Earth's Gravitational Field and Secular Variations in Position, Proceedings from a conference held 26-30 November, 1973 at New South Wales, Sydney Australia, Australian Academy of Science and the International Association of Geodesy.

Papo, H.B. and Perelmuter, A., 1983. Reparametrization of Deformation Analysis, Manuscripta Geodaetica, 8, 41-58.

Pelzer, H., 1974. Zur Behandlung singulärer Ausgleichungsaufgaben, Zeitsschrift für Vermessungswesen, 99, 181-194, 479-488.

Pope, A.J., 1973. The use of the "solution space" in the analysis of geodetic network adjustments, Pres. to the IAG Symp. on Computational methods in Geometric Geodesy, Oxford, 2-8 Sept.

Prescott, 1981. The determination of displacement fields from geodetic data along a strike slip fault, J. Geoph. Res., 86(B7), 6067-6072.

Reilly, W.I., 1981. Complete determination of local crustal deformation from geodetic observations. In: Vyskocil, P., Green, R. and Malzer, H., eds., Recent Crustal Movements 1979, Tectonophysics, 71(1981), 111-123.

Rossikopoulos, D., 2001. Modeling Alternatives in Deformation Measurements, First International Symposium on Robust Statistics and Fuzzy Techniques in Geodesy and GIS, March 12-16, ETH.

Rossikopoulos, D., 2013. Time-depended geodetic networks and the refernce frame definition problem. In: Arabelos, D., Kaltsikis, C., Spatalas, S. and Tziavos, I.N., eds., THALES, Volume in honor of Professor Emeritus Michael E. Contadakis. Publication of Faculty of Rural and Surveying Engineering, Aristotle University of Thessaloniki, 266-287.

Rossikopoulos, D., 2016. Tectonic Geodesy. Measuring the Deformations of the Earth and Constructions (under publication, in Greek), Ziti Publications.

Rossikopoulos, D., Fotiou, A., Livieratos, E. and Baldi, P., 1998. A rigorous analysis of GPS data to detect crustal deformations. Application in the area of the Ionian Sea, Tectonophysics, 294, 271-280.

Rossikopoulos, D. and Fotiou, A., 2001. The Sequential Approach in Geodetic Determination of Crustal Deformations, Poster presented at IAG Scientific Assembly, Vistas for Geodesy in the New Millenium. September 2-7, Budapest, Hungary.

Segall and Matthews, 1988. Displacement Calculations from geodetic data and the testing of geophysical deformation models, J. of Geoph. Res., 93(B12), 14954-14966.

Snay, R.A. and Cline, M.W., 1980. Geodetically derived strain at Shelter Cove, California, Bull. Seism. Soc. Am., 70, 893-902.

Snay, R.A. and Gergen, J.G., 1978. Monitoring regional crustal deformation with horizontal geodetic 
data, Department of Geodetic Science, Report No. 280, The Ohio State University, 87-92.

Snay, R.A., Cline, M.W. and Timmerman, E.L., 1983. Regional Deformation of the Earth Model for the San Diego Region, California, J. of Geoph. Res., 88(B6), 5009-5024.

Teunissen, P.J.G., 1985. Generalized Inverses, Adjustment, the Datum Problem and STransformations. In: Grafarend, E. and Sanso, F., eds., Optimization and Design of Geodetic Networks, Springer Verlag.

van Mierlo, J., 1980. Free network adjustment and S-transformations, In: Deutsche Geod. Komm., Reihe B, 252, 41-54.

van Mierlo, J., 1982. Some aspects of strain analysis by geodetic methods, In: Baarda, W., Anniversary Volume, Geodetic Institute, Technical University of Delft, 506-526.

Vaniček, P., 1975. Vertical crustal movements in Nova Scotia as determined from scattered geodetic relevellings, Tectonophysics, 29, 183-189.

Vaniček, P., Elliot, M.R. and Castle, R.O., 1979. Four-dimensional modeling of recent crustal movements in the area of the Southern California Uplift, Tectonophysics, 52, 287-300.

Welsch, W., 1983. Finite element analysis of strain patterns from geodetic observations across a plate margin, Tectonophysics, 97, 57-71.

Welsch, W.M., 1986. Some aspects of the analysis of geodetic strain observations in kinematic models, Tectonophysics, 130437-458.

Whitten, C.A., 1960. Horizontal movement in earth's crust, J. Geoph. Res., 65, 2839-2844.

Whiten, C.A., 1967. Geodetic networks versus time, Bulletin Géodésique, 84(1), 109-116.

Whitten, C.A. and Claire, C.N., 1960. Analysis of geodetic measurements along the San Andreas fault, Bull. Seismol. Soc. Amer., 50, 404-415.

Wu, J.C., Tang, H.W., Chen, Y.Q. and Li, Y.X., 2006. The current strain distribution in the North China Basin of eastern China by least-squares collocation, J. Geodynamics, 41, 462-470. 